\title{
A Study of the Motion of the Nearby Red Dwarf Lalande $21185^{1}$
}

\author{
NATALIA A. SHAKHT \\ Main Astronomical Observatory of the Russian Academy of Sciences, \\ Pulkovo, St. Petersburg 196140, Russia
}

\begin{abstract}
A series of photographic observations spanning the interval 1962 1991 have been examined in a study of Lalande 21185, suspected to be a double or multiple object. The observed value of secular acceleration corresponds to the radial velocity of this star. The perturbations in proper motion with suspected period of 8 years have not been confirmed.
\end{abstract}

The star Lalande 21185 [R.A. $=11^{h} 00^{m} .6, \quad$ Dec. $=+36^{\circ} 18^{\prime} \quad(1950.0)$, $\mathrm{V}_{\mathrm{r}}=-86.5 \mathrm{~km} \mathrm{~s}^{-1}, \pi_{\mathrm{tr}}=0^{\prime \prime} .396$ (Woolley et al. 1970), $\mathrm{M}_{V}=7^{m} .5, \mathrm{M} 2 \mathrm{~V}$ (Blanco et al. 1968), $M=0.33 \mathcal{M}_{\odot}$ (Lippincot 1960)] is the nearest star to the Sun. It belongs to the Pulkovo program of double and multiple stars as an object with suspected unseen companions. Part of the observational material has been previously studied (Shakht 1972).

Observations of this star have been made since 1962 with the Pulkovo 26-in refractor $(F=10.4 \mathrm{~m}, \mathrm{D}=65 \mathrm{~cm})$. During the first period of observations (19621975) the magnitude difference between the central object and reference stars was corrected by means of an objective grating with $\Delta m=3^{m}$. A short exposure time was used to ensure distinct images; as a result, only a limited number of reference stars were available. Since 1976 a neutral filter was used, and we were able to increase the time of exposure and number of reference stars.

At present we have more than one hundred plates of this star. Recently 93 plates with about 600 exposures obtained over the interval 1962-1991 years were examined.

The treatment was carried out by means of six-constants method using the standard plate with orientations to 1950.0. This method is described by Kisselev (1988) as applied to parallaxes determinations.

As we have included the plates made with the grating in our treatment, only four reference stars were used for this total series. These stars may be found in the list of reference stars for the Lalande 21185 field by Gatewood (1974) under the numbers $8,12,14$, and 15 .

The values of $X, Y$ characterizing the geocentric motion of the object with respect to the standard reference frame were obtained. solved:

In the first stage of treatment the following equations of condition were

$$
\begin{aligned}
& X=C_{x}+\mu_{x} t+\pi_{x} P_{\alpha}+Q_{x} t^{2} \\
& Y=C_{y}+\mu_{y} t+\pi_{y} P_{\delta}+Q_{y} t^{2}
\end{aligned}
$$

\footnotetext{
${ }^{1}$ The author of this paper was unable to attend the Colloquium - eds.
} 
where $(X, Y)$ represent the reduced position of the central star in R.A. and Dec. to the standard plate, $\left(C_{x}, C_{y}\right)$ the errors of the standard plate, $\left(\mu_{x}, \mu_{y}\right)$ and $\left(\pi_{x}, \pi_{y}\right)$ the relative proper motion and annual parallax, $\left(P_{\alpha}, P_{\delta}\right)$ the parallactic factors, and $\left(Q_{x}, Q_{y}\right)$ the quadratic time coefficients, which are equal to half of the observed acceleration; $t$ is counted from a convenient epoch in the middle of the series.

Since the geographic latitude and seeing at Pulkovo did not permit observing this star over a large time interval within the whole year, we did not obtain the parallax with sufficient weight. Moreover, there was a danger of some correlation between parallax and other unknowns.

Therefore we eliminated the parallactic motions by using the catalogue value of parallax and come to the following equations:

$$
\begin{aligned}
X^{\prime} & =C_{x}+\mu_{x} t+Q_{x} t^{2} \\
Y^{\prime} & =C_{y}+\mu_{y} t+Q_{y} t^{2}
\end{aligned}
$$

where $\left(X^{\prime}, Y^{\prime}\right)$ represent the heliocentric positions of the object with respect to the adopted reference frame.

We had also the possibility to study the total proper motion $\bar{\mu}$ of Lalande 21185 by using the value of $L: L=X^{\prime} \sin \psi+Y^{\prime} \cos \psi$, where $\psi$ is a positional angle equal to $\arctan \left(\mu_{x} / \mu_{y}\right)$.

The values of $\left(\mu_{x}, \mu_{y}\right)$ and $\left(X_{x}, Q_{y}\right)$ and total $\bar{\mu}$ and $\bar{Q}$ calculated from $L$ and their mean errors are given in Table 1 . The errors of unit weight (1 plate with 6 exposure) are 0".031 in R.A. and 0".040 in Dec. for this series of plates.

The relative parallax $\pi_{x}$ whose value we have determined in the first stage of treatment was equal to 0.417 with a mean error of 0.009 , but we do not consider this result as a certain one.

TABLE 1. Proper motion and quadratic time coefficients for Lalande 21185

\begin{tabular}{|cccccc|}
\hline$\mu_{d} \cos \delta / \mathrm{yr}$ & $\mu_{\delta} / \mathrm{yr}$ & $\bar{\mu} / \mathrm{yr}$ & $Q_{x} / \mathrm{yr}^{2}$ & $\bar{Q}_{y} / \mathrm{yr}^{2}$ & $\bar{Q} / \mathrm{yr}^{2}$ \\
\hline$-0^{\prime \prime} .5519$ & $-4^{\prime \prime} .7616$ & $-4^{\prime \prime} .7939$ & $-0^{\prime \prime} .000085$ & $-0^{\prime \prime} .000144$ & $-0^{\prime \prime} .000157$ \\
\pm 0.0004 & \pm 0.0006 & \pm 0.0006 & \pm 0.000058 & \pm 0.000074 & \pm 0.000075 \\
\hline
\end{tabular}

We do not consider the value of $Q_{x}$ as definitive because of the relatively short time span (29 years) used for its determination.

The results of determinations of $\bar{Q}$ and $Q_{\nu}$ are in agreement with $\bar{Q}=0^{\prime \prime} .00020 \pm 0^{\prime \prime} .00009$ and of $\mu$ values for the middle of the series of $1962-$ 1975 and 1976-1991 according to the formula:

$$
Q=\dot{\mu} / 2=\frac{\mu_{2}-\mu_{1}}{2\left(t_{2}-t_{1}\right)}
$$

The results of these observations are in agreement with the theorectical value of perspective effect $Q\left(-0^{\prime \prime} .00017\right)$ which is calculated by means of the formula: 


$$
Q=-1.02 \mu \pi V_{\mathrm{r}} 10^{-6} / \mathrm{yr}^{-2} .
$$

Thus the radial velocity which may be determined from secular acceleration $\left(V_{r, \text { dynamic }}\right)$ must not differ essentially from $\left(V_{r, \text { spectroscopic }}\right)$ and the red shift is not revealed. The absence of this effect is in agreement with the nature of this red dwarf.

Our analysis of the series 1962-1972 (Shakht 1972) and the treatment of all data 1962-1991 did not show the periodic perturbations in the proper motion of Lalande 21185 which were discussed by Lippincott (1960). But there are some ambiguities in the residuals, and we intend to make a more detailed study of them.

I would like to thank A. A. Kisselev for his attention to this work and useful discussion.

\section{REFERENCES}

Blanco, V.M., Demers, S., Douglass, G.G., \& Fitzgerald, M.P. 1968, "Photoelectric Catalogue: Magnitudes and Colors of Stars in the U, B, V and $U_{C}, B, V$ Systems", Publ. U.S. Naval Obs. 21

Gatewood, G. 1974, $A J, 79,52$

Kisselev, A.A. 1988, The Theoretical Foundations of Photographic Astrometry, Publ. "Nauka" Moscow USSR (in Russian)

Lippincott, S.L. 1960, $A J, 65,445$

Shakht, N.A. 1972, Astr. Circ. USSR, 736, 5 (in Russian).

Woolley, R., Epps, E.A., Penston, M.J., \& Pocock, S.B. 1970 Catalogue of Stars within 25 parsec of the Sun, Royal Obs. Annal 5 